\title{
Defect modes in microring resonator arrays fabricated in silicon-on-insulator technology
}

\author{
Landobasa Y. M. Tobing ${ }^{1}$, Pieter Dumon ${ }^{2}$, Roel Baets ${ }^{2}$, and Mee-Koy Chin ${ }^{1 *}$ \\ ${ }^{1}$ Nanyang Technological University, Singapore \\ ${ }^{2}$ University of Ghent, Belgium \\ *E-mail: emkchin@ntu.edu.sg
}

\begin{abstract}
We show experimentally the existence of defect modes in mutually coupled microring resonator array fabricated in silicon-on-insulator technology. The movements of donor-like and acceptor-like modes are demonstrated for various defect lengths and in a good agreement with earlier theoretical prediction.
\end{abstract}

Microring resonator is an integrated-optic form of FabryPerot etalon that is capable of realizing various active and passive functionalities, such as filters, lasers, sensors, and switches. These functionalities can be further extended by integrating these resonators in array geometries for slow light structure [1, 2] and high order filters [3-5]. In periodic array geometries consisting of identical rings, there exists a frequency band within which the light is not allowed to propagate inside the structure. This is called a photonic bandgap (PBG) mechanism which bears remarkable resemblance to the electronic bandgap in solid state physics. Similar to its electronic counterpart, introducing irregularities (or defect) inside the structure may excite a defect mode where light is localized near that defect. The concept of defect mode is not new and has been demonstrated in conventional photonic bandgap structures [6], where usually the defect is in the form of a hole of different size or a dislocation [7, 8]. However, the concept of defect modes in periodic arrays of microring resonators has not been extensively explored. This structure is somewhat different because of the hybrid properties of photonic bandgap and resonators. Previously we have theoretically demonstrated the existence of defect modes in such hybrid structures [9], and in this paper we present for the first time the experimental realization of the defect modes, verifying the existence of acceptor and donor modes, in microring resonator arrays fabricated in silicon-on-insulator technology.

As shown in Fig. 1, our configuration consists of series of mutually coupled microring resonators with a defect ring at the center of the structure. The defect in our configuration takes the form of a ring with different size. The relative size of the defect ring from the regular rings $\left(k_{\mathrm{D}}=L_{\mathrm{DEFECT}} / L_{\mathrm{CAV}}\right)$ determines the type of 'doping' in the structure. The introduction of a larger (smaller) defect ring is analogous to doping a donor (acceptor) energy level in electronic band structure.

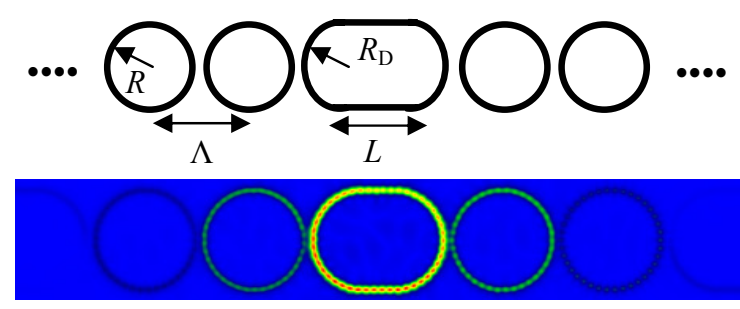

Fig. 1. (Top) The schematic of a defect mode configuration in a ring resonator periodic array, and (bottom) the FDTD simulated field distribution in and near the defect ring when a Gaussian pulse is injected from an input waveguide.

The detailed model for such an optical structure has been presented in [9]. In the absence of the defect ring $\left(k_{\mathrm{D}}=1\right)$, it is shown that such a configuration exhibits a photonic band structure given by the dispersion characteristics

$$
\cos k \Lambda=\left(\frac{1}{t}\right) \sin \left(\frac{\omega n_{\mathrm{eff}} L_{C A V}}{2 \mathrm{c}}\right)
$$

where $t$ is the amplitude coupling coefficient between adjacent rings, $\mathrm{c}$ is the speed of light in vacuum, $L_{\mathrm{CAV}}$ is the ring circumference, $n_{\text {eff }}$ is the effective index of the waveguide, $k$ is the Bloch wavevector, and $\Lambda=L_{\mathrm{CAV}} / 2$ is the period of the unit cell. This type of periodic structure is sometimes referred to as coupled resonator optical waveguide (CROW).

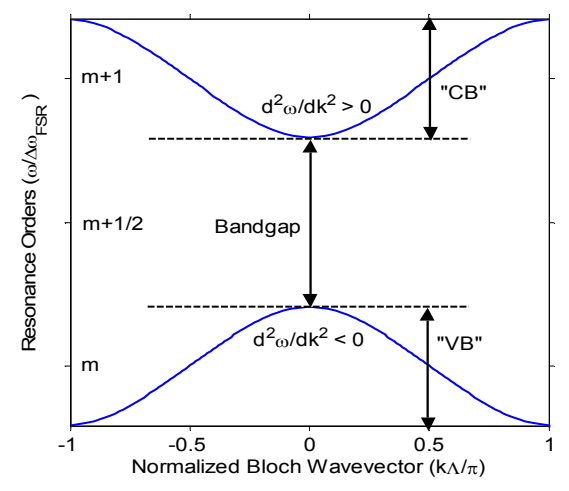

Fig. 2. The band structure of CROW. 
Fig. 2 shows the dispersion relation of a typical CROW structure of a particular resonances orders $\left(m=\omega / \Delta \omega_{\mathrm{FSR}}\right)$. When the resonators are on resonance, the cavity modes from individual rings interact and create a continuous band centralized at resonance $\left(\omega / \Delta \omega_{\mathrm{FSR}}=m\right)$ and the light propagation is allowed in the structure with the group velocity proportional to the coupling factor $(t)$. When the resonators are off resonance, the Bloch wavevector $k$ becomes imaginary and a photonic bandgap is formed centered at the anti-resonance frequency $\left(\omega / \Delta \omega_{\mathrm{FSR}}=m+1 / 2\right)$. The light then is reflected from the structure in the bandgap region. The difference between this hybrid structure and the conventional PBG is the fact that the bandgap develops faster because the resonator resonantly enhances the reflection in each cells. Thus, fewer numbers of cells are needed to have the same bandgap properties.

Similar to in solid state electronics, the curvature of the continuous band $\left(d^{2} \omega / d k^{2}\right)$ determines the type of the band in that the positive (negative) curvature is analogous to the conduction (valence) band in electronics. As one can see in Fig. 2, there is always a pair of conduction and valence bands for every resonance order. If one introduces a defect ring inside the structures, then it is possible to have strong light localization in the vicinity of such defect. The lower panel of Fig. 1 shows the finite difference time domain simulation of the defect mode field. The light is strongly localized near the defect ring and the amplitude of the circulating light decays with distance from the defect. This is because the defect modes are located within the bandgap and has an imaginary Bloch wavevector along the structure (see Fig. 2). The type of defect mode depends on the type of 'doping' that is introduced. If the defect ring is a smaller ring, then an acceptor-like mode is excited from the valence band (VB). Similarly, if the defect ring is larger, then a donor-like mode will be excited from the conduction band (CB). In the finite structure, the $\mathrm{Q}$ factor of the defect resonance depends on its relative location from the continuous bands. The $\mathrm{Q}$ is higher when the resonance is farther from the continuous bands due to stronger reflection from the periodic sub-arrays. The reflection is nearly $100 \%$ at the center of the bandgap for finite structures, thus theoretically can give near infinite $\mathrm{Q}$ factors. However, in the presence of loss the amplitude of high $\mathrm{Q}$ resonance is quickly degraded.

In order to experimentally demonstrate the existence and dispersion of these defect modes, we designed a set of mutually coupled microring arrays, each with 4 identical rings and one defect ring at the center (see Fig. 3). The number of rings is maintained low to limit the effect of loss in the measurement. The devices were fabricated using deepUV (DUV) CMOS process in silicon-on-insulator (SOI) technology [10]. The waveguide width is $450 \mathrm{~nm}$ and the silicon core thickness is $200 \mathrm{~nm}$. The ring radius is $8 \mu \mathrm{m}$. The couplings between the rings and between the input waveguide and the ring are introduced by racetrack coupling of length $L_{\mathrm{C}}=6 \mu \mathrm{m}$, which is designed to give a fairly large coupling in order to ease the defect mode excitation. Finally, to demonstrate the existence of donor and acceptor modes, we varied the defect ring sizes such that the defect parameter ranges from $k_{\mathrm{D}}=0.7$ to $k_{\mathrm{D}}=2$. The defect sizes are varied by adjusting the extension length $(L)$ and defect radius $\left(R_{\mathrm{D}}\right)$ in the defect ring, as shown in Fig. 3.

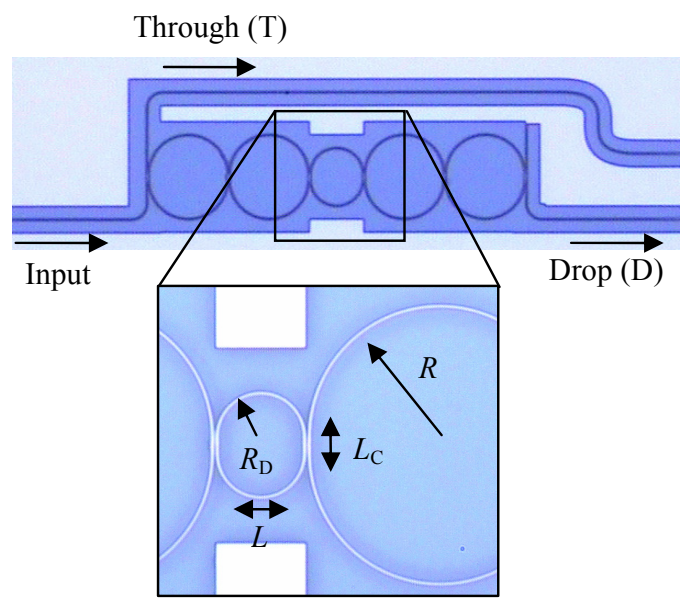

Fig. 3. The fabricated devices showing the defect ring in the center of the 4 ring array coupled to two bus waveguides. The defect parameter $k_{\mathrm{D}}$ is controlled by varying $L$ and $R_{\mathrm{D}}$

In the measurements, the device is excited with a broadband source, and coupling into the optical waveguide is facilitated via a second order grating that has been integrated with the device. The fiber is butt-coupled to the grating $10^{\circ}$ off vertical. The coupling efficiency has a Gaussian spectral profile with a bandwidth of about $30 \mathrm{~nm}$. The output is passed through a 90:10 splitter, where $10 \%$ power goes to a fiber power meter (FPM) for alignment purpose and the rest goes to an optical spectrum analyzer (OSA) for normalization of the spectrum.

First, we show the traces of donor-like modes in Fig. 4, which plots the measured Drop transmissions for increasing defect size. The defect modes are the resonances located within the bandgap region. For clarity the transmissions for different defect modes are offset by $-20 \mathrm{~dB}$. As the insertion loss is still high, to make the results clearer, we filtered out the underlying noises by setting a lower transmission threshold of $-15 \mathrm{~dB}$. The photonic bandgap is then identified from the low and flat transmission band as indicated in the shaded gray region. The continuous transmission region with shorter wavelength is the conduction band (CB) and that with the longer wavelength is the valence band (VB). When the defect ring is only slightly different from the other rings $\left(k_{\mathrm{D}} \sim 1\right)$, one can observe a defect mode (labeled 1) very close to the conduction band edge. When $k_{\mathrm{D}}$ is progressively increased, mode 1 begins to move from $\mathrm{CB}$ to $\mathrm{VB}$. At $k_{\mathrm{D}} \sim 1.7$, a second donor mode (mode 2 ) emerges near the $\mathrm{CB}$ edge, and it moves in the same direction along with mode 1. Finally, in the last graph where $k_{\mathrm{D}} \sim 2$ we see yet another mode (mode 3 ) being excited and emerging near the CB. 


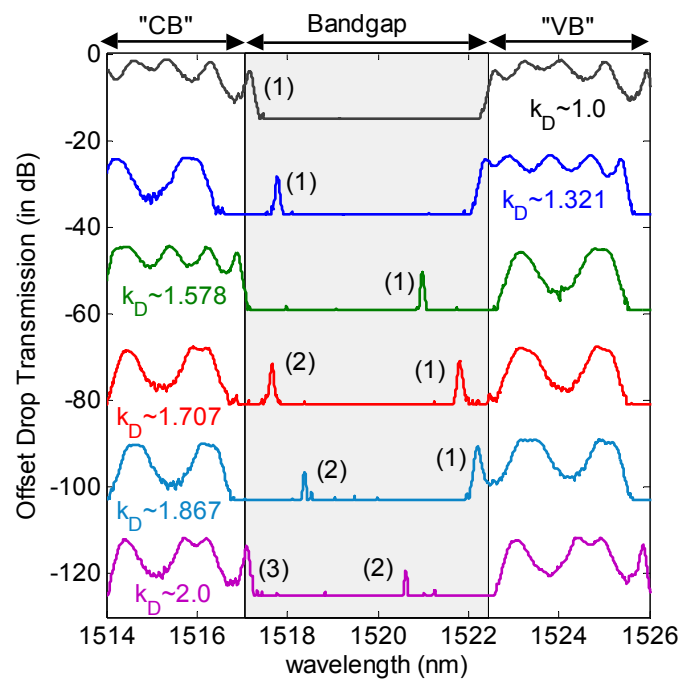

Fig. 4. Traces of how donor modes shift in wavelength with increasing defect size.

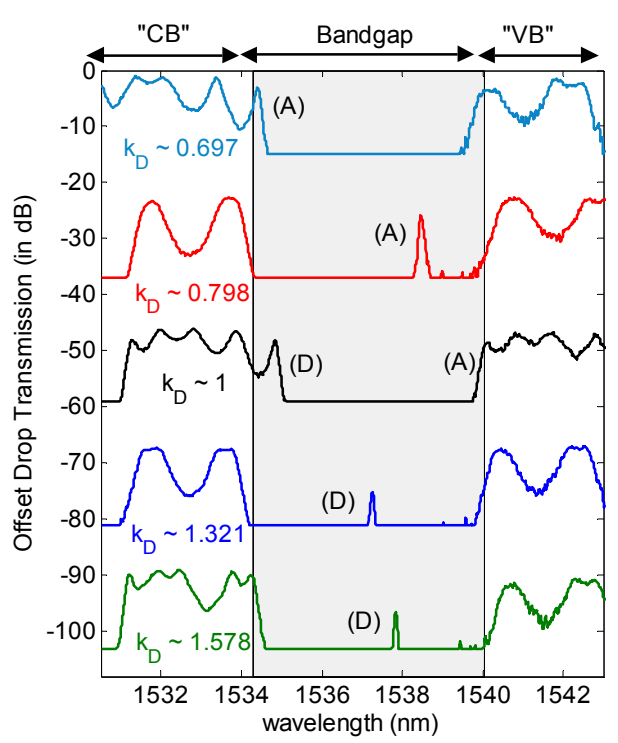

Fig. 5. Traces of acceptor (A) and donor (D) modes with varying $k_{\mathrm{D}}$.

Finally, we show the traces of both acceptor-like modes and donor-like modes together in Fig. 5, which plots the Drop transmissions from smaller $\left(k_{\mathrm{D}}<1\right)$ to larger defect ring size $\left(k_{\mathrm{D}}>1\right)$. In solid state physics, an acceptor mode moves from $\mathrm{VB}$ to $\mathrm{CB}$ when the acceptor level is increased. By analogy, in our photonic structure, the acceptor mode should move to the shorter wavelength with decreasing defect size $\left(k_{\mathrm{D}}<1\right)$. Note that this is to be distinguished from the movement of the two donor modes in Fig. 4 that occurs for $k_{\mathrm{D}}>1$. When $k_{\mathrm{D}} \sim 1$, there exists two modes at the CB and VB edges, and we address them as acceptor mode (A) and donor mode (D), respectively. For $k_{\mathrm{D}}<1$ (the smaller defect rings), mode (A) shifts to shorter wavelength while mode (D) is no longer observed. The opposite occurs for $k_{\mathrm{D}}>1$ (the larger defect rings).

In conclusion, we have demonstrated experimentally the existence of defect modes in a ring-resonator array hybrid structure. Furthermore, by observing the opposite movement of the defect modes for larger and smaller defect ring sizes, respectively, we confirm the existence of donor mode (D) and acceptor mode (A) as we have predicted in Ref. [9]. In addition, we have verified the unique ability of the hybrid resonator photonic bandgap structure in generating the photonic bandgap with very few number of unit cells, as is apparent from our current fabricated devices that have only 4 unit cells. We observe that the Q of the defect resonance is highest when it is located near the center of the photonic bandgap, but the amplitude is also smallest because of the accentuated effect of loss. In general, the $Q$ can be significantly enhanced by slightly increasing the number of unit cells and reducing all the coupling coefficients.

\section{ACKNOWLEDGMENT}

The authors would like to thank Jean Marc Fedelli for fabricating the devices in CEA-LETI under the e-PIXnet platform

\section{REFERENCES}

[1] A. Yariv, Y. Xu, R. K. Lee, and A. Scherer, "Coupled-resonator optical waveguide: a proposal and analysis," Opt. Lett. 24, 711-713 (1999).

[2] J. K. S. Poon, J. Scheuer, S. Mookherjea, G. T. Paloczi, Y. Huang, and A. Yariv, "Matrix analysis of microring coupled-resonator optical waveguides," Opt. Exp. 12, 90-103 (2004).

[3] G. Griffel, "Synthesis of optical filters using ring resonator arrays," IEEE Photon. Technol. Lett. 12, 810-812 (2000).

[4] J. V. Hryniewicz, P. P. Absil, B. E. Little, R. A. Wilson, and P-T. Ho, "Higher order filter response in coupled microring resonators," IEEE Photon. Technol. Lett. 12, 320-322 (2000).

[5] B.E. Little, S. T. Chu, P. P. Absil, J. V. Hryniewicz, F. G. Johnson, F. Seiferth, D. Gill, V.Van, O.King, and M. Trakalo, "Very high-order microring resonator filters for WDM applications," IEEE Photon. Technol. Lett. 16,.2263-2265 (2004).

[6] E. Yablonovitch, "Inhibited spontaneous emission in solid-state physics and electronics," Phys. Rev. Lett. 58, 2059-2062 (1987).

[7] S. Fan, J. N. Winn, A. Devenyi, J. C. Chen, R. D. Meade, and J. D. Joannopoulos, "Guided and defect modes in periodic dielectric waveguides,” J. Opt. Soc. Am. B 12, 1267 (1995).

[8] K. Sakoda and H. Shiroma, "Numerical method for localized defect modes in photonic lattices," Phys. Rev. B 56, 4830 (1997).

[9] Y. Landobasa and M. K. Chin, "Defect modes in micro-ring resonator arrays," Opt. Express 13, 7800-7815 (2005).

[10]W. Bogaerts, R. Baets, P. Dumon, V. Wiaux, S. Beckx, D. Taillaert, B. Luyssaert, J. Van Campenhout, P. Bienstman and D. Van Thourhout, "Nanophotonic waveguides in Silicon-on-Insulator with CMOS Technology,” IEEE J. Lightwave Technol. 23, 401-412 (2005). 